\title{
The relationship between adenosine deaminase and heart rate-corrected QT interval in type 2 diabetic patients
}

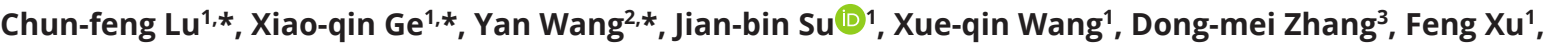 \\ Wang-shu Liu ${ }^{1}$ and Min Su ${ }^{4}$ \\ ${ }^{1}$ Department of Endocrinology, Affiliated Hospital 2 of Nantong University and First People's Hospital of Nantong City, Nantong, China \\ ${ }^{2}$ Department of Geriatrics, Affiliated Hospital 2 of Nantong University and First People's Hospital of Nantong City, Nantong, China \\ ${ }^{3}$ Clinical Medicine Research Center, Affiliated Hospital 2 of Nantong University and First People's Hospital of Nantong City, Nantong, China \\ ${ }^{4}$ Department of Endocrinology, Nantong Hospital of Traditional Chinese Medicine, Nantong Hospital Affiliated to Nanjing University of Chinese Medicine, \\ Nantong, China
}

Correspondence should be addressed to J Su or X Wang: sujbzjx@163.com or wangxueqin108@163.com

*(C Lu, X Ge and Y Wang contributed equally to this work)

\begin{abstract}
Background: Prolonged heart rate-corrected QT (QTc) interval may reflect poor prognosis of patients with type 2 diabetes (T2D). Serum adenosine deaminase (ADA) levels are related to hyperglycemia, insulin resistance (IR) and inflammation, which may participate in diabetic complications. We investigated the association of serum ADA levels with prolonged QTc interval in a large-scale sample of patients with T2D.

Methods: In this cross-sectional study, a total of 492 patients with T2D were recruited. Serum ADA levels were determined by venous blood during fasting. QTc interval was estimated from resting 12-lead ECGs, and prolonged QTc interval was defined as QTc > 440 ms. Results: In this study, the prevalence of prolonged QTc interval was $22.8 \%$. Serum ADA levels were positively associated with QTc interval $(r=0.324, P<0.0001)$. The proportion of participants with prolonged QTC interval increased significantly from $9.2 \%$ in the first tertile (T1) to $24.7 \%$ in the second tertile (T2) and $39.0 \%$ in the third tertile (T3) of ADA ( $P$ for trend $<0.001$ ). After adjusting for other possible risk factors by multiple linear regression analysis, serum ADA level was still significantly associated with QTc interval $(\beta=0.217, t=3.400, P<0.01)$. Multivariate logistic regression analysis showed that female (OR 5.084, CI 2.379-10.864, $P<0.001$ ), insulin-sensitizers treatment (OR 4.229, Cl 1.290-13.860, $P=0.017$ ) and ADA (OR 1.212, CI 1.094-1.343, $P<0.001$ ) were independent contributors to prolonged QTc interval. Conclusions: Serum ADA levels were independently associated with prolonged QTc interval in patients with T2D.
\end{abstract}

Endocrine Connections (2021) 10, 894-901

\section{Introduction}

Diabetes and its complications have posed a major threat to global health, and over $90 \%$ of these cases are type 2 diabetes (T2D) (1). According to a report of the International Diabetes Federation (IDF), about 5.0 million people died due to diabetes and its complications in 2015 (2). Among them, cardiovascular complications are the leading cause of diabetes-related mortality (3). Therefore, early identification and intervention of cardiovascular https://ec.bioscientifica.com https://doi.org/10.1530/EC-21-0199

(c) 2021 The authors Published by Bioscientifica Ltd



This work is licensed under a Creative Commons Attribution-NonCommercial 4.0 International License. ded from Bioscientifica.com at 04/26/2023 10:29:17AM 
complications are the focus of T2D management. In the absence of overt cardiovascular disease, the assessment of heart rate-corrected QT (QTc) interval may have a potential role in screening for cardiovascular complications (4). Prolonged QTC interval may be a surrogate marker for subclinical atherosclerosis and diabetic cardiomyopathy (5). In addition, prolonged QTc interval is a predictor of cardiovascular mortality and all-cause mortality in patients with T2D (6). Even worse, the prevalence of prolonged QTc interval in patients with T2D is as high as 30\% (7). Therefore, it is of great clinical significance to fully assess the risk factors for prolonged QTc interval in T2D patients.

Adenosine deaminase (ADA), abundantly expressed in various cells and tissues, regulates serum adenosine concentration through catalyzing the irreversible deamination of adenosine to inosine (8). Growing evidence has shown that serum ADA levels are significantly increased in patients with diabetes and positively associated with fasting plasma glucose (FPG), glycated hemoglobin (HbA1c) and insulin resistance (IR) $(9,10,11)$. A large number of studies have shown that adenosine can play a role in cardiac protection by dilating the coronary artery, scavenging oxygen free radicals, inhibiting platelet activation and maintaining cholesterol homeostasis $(12,13)$. Thus, elevated serum ADA levels in T2D may be associated with myocardial ischemia. Coincidentally, Yu et al. have found that serum ADA levels are associated with the severity of atherosclerosis (14). A cross-sectional study of 501 patients with T2D exhibits that hyperglycemia and myocardial ischemia are important contributors to prolonged QTc (15). Hereby, we hypothesized that increased serum ADA levels possibly contribute to prolonged QTc interval or at least reflect an increased risk of prolonged QTc interval in patients with T2D. However, to our knowledge, no studies have investigated the relationship between serum ADA levels and QTc interval in T2D patients.

Therefore, the present study was designed to explore the relationship between serum ADA levels and QTc interval in a Chinese population with T2D.

\section{Methods}

\section{Study design and participants}

This was an observational cross-sectional study, and a total of 492 patients with T2D were enrolled at the inpatient department of the Second Affiliated Hospital of Nantong University between July 2020 and December 2020. Patients with T2D diagnosed based on the statement of the
American Diabetes Association in 2011 were eligible for the present study (16). The exclusion criteria were: (1) type 1 diabetes (T1D); (2) cardiac disease, such as atrial fibrillation, atrial flutter, cardiac conduction block, heart valve disease, myocardial infarction and so on; (3) previous and current malignant tumors; (4) chronic hepatic disease; (5) acute diabetic complications, such as diabetic ketoacidosis; (6) previous use of drugs that affect the QT interval, such as a tricyclic antidepressant; and (7) concomitant with hyperthyroidism, hypothyroidism and other endocrine diseases. The study completely adhered to the Declaration of Helsinki, and the written informed consent were signed by each participant. The study procedures were approved by the medical research ethics committee of the Second Affiliated Hospital of Nantong University.

\section{Basic data collection}

During enrollment, all patients were interviewed to record information including age, sex, past illness and medical history, and routine physical examinations were performed by experienced physicians. BMI was calculated as the weight (kilogram)/height (meter) squared. After resting for at least $30 \mathrm{~min}$, blood pressure was measured three times by a standard mercury sphygmomanometer, and then the average was recorded for further analysis.

\section{QTc interval analysis}

After resting for $30 \mathrm{~min}$, all participants underwent a standard resting 12-lead ECGs (FX-7402, CardiMax, FuTian Beijing Ltd., China) in a quiet and secluded room, and each ECG was recorded on a standard paper with a waveformsamplitude of $10 \mathrm{~mm} / \mathrm{mV}$ and a traveling-rate of $25 \mathrm{~mm} / \mathrm{s}$. Each ECG report was interpreted by the Nalong ECG information management system (Nalong Sci \& Tech Co Ltd., China), and the QTc intervals were collected directly from the system. A prolonged QTc interval was defined as a QTc interval of more than $440 \mathrm{~ms}$ (17).

\section{Laboratory examination}

When the participants were fasting for at least $8 \mathrm{~h}$ in the morning, venous blood samples were drawn to measure laboratory parameters, and fresh first-void morning urine samples were collected for measurement of urinary albumin and urinary creatinine. An automated biochemical analyzer (Model 7600, Hitachi) was used to measure serum ADA, triglyceride (TG), total cholesterol (TC), low-density lipoprotein cholesterol (LDL-c), high-density lipoprotein

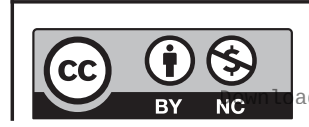

This work is licensed under a Creative Commons Attribution-NonCommercial 4.0 International License. ded from Bioscientifica.com at 04/26/2023 10:29:17AM 
cholesterol (HDL-c), blood urea nitrogen (BUN), creatinine (Cr), cystatin $\mathrm{C}$ and uric acid (UA) levels. An ion exchangebased HPLC method in a hemoglobin analysis system (D-10, Bio-Rad) was used to measure HbA1c levels. The equation of urinary albumin level/urinary creatinine level was calculated as the urinary albumin/creatinine ratio (UACR). The estimated glomerular filtration rate (eGFR) was calculated based on the Chronic Kidney Disease Epidemiology Collaboration (CKD-EPI) creatininecystatin C equation (2012) (18).

\section{Statistical analyses}

Clinical variables were calculated for the total subjects and across the tertiles of serum ADA levels. The mean \pm S.D., median (25 and $75 \%$ interquartile), and frequency (percentage) were adopted to represented continuous variables with normal distributions, continuous variables with skewed distributions and categorical variables, respectively. The differences in normally distributed data, skewed data and categorical data between the ADA tertiles were compared by one-way ANOVA, the Kruskal-Wallis test and the chi-square test, respectively. The correlation between serum ADA and QTc interval was analyzed by Spearman's bivariate correlation analysis. Multiple linear regression analysis was conducted to investigate the independent association of serum ADA level with the QTc interval. Furthermore, univariate and multivariate logistic regression analyses were constructed to evaluate the impact of ADA and other risk factors on prolonged QTc, and the corresponding odds ratios (ORs) and 95\% CIs were provided. All analyses were conducted using SPSS statistical software 18.0 (IBM SPSS Inc.). A value of $P<0.05$ was considered statistically significant.

\section{Results}

\section{Basic characteristics}

The clinical characteristics of the total participants and the three subgroups according to ADA tertiles are shown in Table 1. Among the enrolled 492 patients with T2D, the mean QTc interval was $422.84 \pm 25.75 \mathrm{~ms}$, and the prevalence of prolonged QTc was $22.8 \%$. From the first tertile to the third tertile of ADA, the QTc interval significantly increased from $415.12 \pm 21.00$ to $421.98 \pm 27.49$ and $434.35 \pm 25.34 \mathrm{~ms}$, and the corresponding proportion of patients with prolonged QTc interval significantly increased from 9.2 to $24.7 \%$ and $39.0 \%$, respectively.
As the ADA tertile increased, the percentage of females, age, diabetes duration, systolic blood pressure (SBP), percentage of patients using insulin, HbA1c and UACR level increased, whereas the percentage of patients using metformin and eGFR decreased $(P$ for trend $<0.05)$. However, BMI, diastolic blood pressure (DBP), use of other antihyperglycemic treatments, antihypertensive treatments, statin medications, lipid profile and UA level did not show any difference among the tertiles of ADA levels ( $P$ for trend $>0.05$ ).

\section{Relationship between QTc interval and ADA}

The correlation between QTc interval and ADA is shown in Fig. 1. The QTc interval was significantly and positively related to serum ADA level $(r=0.324, P<0.001)$.

\section{Multiple linear regression models displayed independent associations of ADA with QTc interval}

As illustrated in the Table 2, serum ADA level was significantly associated with QTc interval $(\beta=0.336$, $t=7.892, P<0.001$, adjusted $\left.R^{2}=0.113\right)$. The adjusted $R^{2}$ was gradually increased along with adding the other clinical covariates in each model. In the fully adjusted model 3, serum ADA level was still significantly associated with QTc interval ( $\beta=0.217, t=3.400, P<0.01$, adjusted $R^{2}=0.249$ ).

\section{Univariate and multivariate analysis explored relationships between multiple risk factors and prolonged QTc interval}

In Table 3, the univariate analysis showed that prolonged QTc interval was closely associated with age (OR 1.209, CI 1.011-1.048, $P=0.002$ ), female (OR 3.396, CI 2.188-5.270, $P<0.001$ ), diabetes duration (OR 1.039, CI 1.012-1.066, $P=0.004)$, SBP (OR 1.013, CI 1.002-1.024, $P=0.022)$, insulin treatment (OR 1.845, CI 1.178-2.889, $P=0.007$ ), insulinsensitizers treatment (OR 2.357, CI 1.133-4.905, $P=0.022$ ), HbA1c (OR 1.173, CI 1.062-1.295, $P=0.002$ ), UACR (OR 1.001, CI 1.000-1.001, $P=0.006$ ) and ADA (OR 1.224, CI 1.153-1.299, $P<0.001)$. Prolonged QTc interval had no concern with BMI, other antihyperglycemic treatments, antihypertensive treatments, statin medications, UA and eGFR. Multivariate logistic regression analysis showed that female (OR 5.084, CI 2.379-10.864, $P<0.001$ ), insulin-sensitizers treatment (OR 4.229, CI 1.290-13.860, $P=0.017$ ) and ADA (OR 1.212, CI 1.094-1.343,

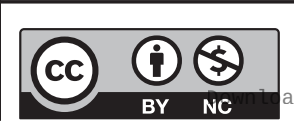

This work is licensed under a Creative Commons Attribution-NonCommercial 4.0 International License. ded from Bioscientifica.com at 04/26/2023 10:29:17AM 
Table 1 Clinical characteristics of the participants.

\begin{tabular}{l}
\hline Variables \\
\hline ADA (U/L) \\
$n$ \\
Age (years) \\
Male, $n$ (\%) \\
Diabetes duration (years) \\
BMI (kg/m²) \\
SBP (mmHg) \\
DBP (mmHg) \\
Antidiabetic treatment \\
Insulin treatment, $n(\%)$ \\
$\quad$ Metformin, $n$ (\%) \\
Acarbose, $n(\%)$ \\
Insulin secretagogues, \\
$\quad n(\%)$ \\
Insulin sensitizers, $n(\%)$ \\
DPP-4 inhibitors, $n(\%)$ \\
Antihypertensive \\
treatments \\
$\quad$ CCB, $n(\%)$ \\
ARB, $n(\%)$ \\
Diuretics, $n(\%)$ \\
Statin medications, $n(\%)$ \\
HbA1c (\%) \\
TG (mmol/L) \\
TC (mmol/L) \\
HDL-c (mmol/L) \\
LDL-c (mmol/L) \\
UA (umol/L) \\
eGFR (mL/min/1.73m²) \\
UACR (mg/g) \\
QTC (ms) \\
Prolonged QTc, $n(\%)$ \\
\end{tabular}

\begin{tabular}{c}
\hline Total \\
\hline $10(9-13)$ \\
492 \\
$57.51 \pm 12.66$ \\
$293(59.6)$ \\
$7(1-10)$ \\
$25.79 \pm 4.07$ \\
$132(122-145)$ \\
$80(74-86)$
\end{tabular}

135 (27.4)

206 (41.9)

47 (9.6)

$155(31.5)$

$33(6.7)$

$21(4.3)$

$113(23.0)$
$99(20.1)$
$34(6.9)$
$23(4.7)$
$9.17 \pm 2.11$
$1.54(1.04-2.55)$
$4.41(3.72-5.12)$
$1.14(0.98-1.35)$
$2.79 \pm 0.92$
$299.0(237.3-376.0)$
$108.05 \pm 28.81$
$15.2(7.8-54.5)$
$422.84 \pm 25.75$
$112(22.8)$

\begin{tabular}{c}
\hline $\mathbf{T 1}$ \\
\hline$<10$ \\
185 \\
$54.28 \pm 11.51$ \\
$132(71.4)$ \\
$4(1-10)$ \\
$25.54 \pm 4.05$ \\
$130(120-141)$ \\
$79(73-86)$
\end{tabular}

35 (18.9)

84 (45.4)

$17(9.2)$

$58(31.4)$

$11(5.9)$

9 (4.9)

$31(16.8)$
$34(18.4)$
$13(7.0)$
$4(2.2)$
$8.63 \pm 1.95$
$1.58(1.08-2.44)$
$4.37(3.72-5.08)$
$1.15(1.01-1.31)$
$2.83 \pm 0.91$
$294.0(239.5-376.5)$
$115.51 \pm 23.04$
$10.4(5.90-31.6)$
$415.12 \pm 21.00$
$17(9.2)$

\begin{tabular}{c}
\hline $\mathbf{T 2}$ \\
\hline $10-12$ \\
170 \\
$57.92 \pm 12.75$ \\
$97(57.1)$ \\
$8(2-10)$ \\
$25.59 \pm 3.56$ \\
$132(122-144)$ \\
$80(74-86)$
\end{tabular}

49 (28.8)

77 (45.3)

$18(10.6)$

54 (31.8)

$17(10.0)$

$6(3.5)$

$43(25.3)$
$36(21.2)$
$7(4.1)$
$12(7.1)$
$9.22 \pm 2.12$
$1.46(0.92-2.36)$
$4.45(3.62-5.19)$
$1.15(1.02-1.41)$
$2.84 \pm 0.95$
$294.5(233.8-345.3)$
$107.36 \pm 27.94$
$16.0(9.3-58.6)$
$421.98 \pm 27.49$
$42(24.7)$

\begin{tabular}{c}
\hline $\mathbf{T 3}$ \\
\hline$>12$ \\
137 \\
$61.37 \pm 12.98$ \\
$64(46.7)$
\end{tabular}

$8(2-18)$

$26.33 \pm 4.57$

$135(126-150)$

$82(75-88)$

$51(37.2)$

45 (33.8)

$12(8.8)$

$43(31.4)$

$5(3.6)$

$6(4.4)$

29 (28.5)

$29(21.2)$

$14(10.2)$

7 (5.1)

$9.83 \pm 2.14$

$1.79(1.14-3.46)$

$4.40(3.80-5.25)$

$1.10(0.93-1.34)$

$2.67 \pm 0.88$

308.0 (239.0-404.5)

$98.74 \pm 33.86$

$22.6(10.4-115.4)$

$434.35 \pm 25.34$

$53(39.0)$

\begin{tabular}{c}
$P$ for trend \\
\hline \\
$<0.001$ \\
$<0.001$ \\
0.023 \\
0.302 \\
0.011 \\
0.231 \\
\\
0.001 \\
0.042 \\
0.844 \\
0.996 \\
\\
0.076 \\
0.822 \\
\\
0.034 \\
0.755 \\
0.111 \\
0.089 \\
$<0.001$ \\
0.063 \\
0.796 \\
0.091 \\
0.229 \\
0.084 \\
$<0.001$ \\
$<0.001$ \\
$<0.001$ \\
$<0.001$ \\
\hline
\end{tabular}

Normally distributed values in the table are given as the mean \pm s.D., skewed distributed values are given as the median ( 25 and $75 \%$ interquartiles), and categorical variables are given as frequency (percentage). ANOVA, the Kruskal-Wallis test and the Chi squared test were conducted to determine $P$ values for normally distributed continuous variables, skewed continuous variables and categorical variables, respectively.

ADA, adenosine deaminase; ARB, angiotensin receptor blockers; CCB, calcium channel blockers; DPP-4 inhibitors, dipeptidyl peptidase-4 inhibitors; eGFR, estimated glomerular filtration rate; HbA1c, glycosylated hemoglobin A1c; HDL-c, high density lipoprotein cholesterol; LDL-c, low density lipoprotein cholesterol; QTC, heart rate-corrected QT interval; SBP/DBP, systolic/diastolic blood pressure; TC, total cholesterol; TG, triglyceride; UA, uric acid; UACR, urinary albumin creatinine ratio.

$P<0.001)$ were significantly correlating with prolonged QTc (Nagelkerke $\left.R^{2}=0.371\right)$.

\section{Discussion}

In the present study, we evaluated the associations between serum ADA levels and QTc interval in a large cohort of Chinese patients with T2D. The main findings are as follows: first, $22.8 \%(n=112)$ of the enrolled patients presented with prolonged QTc interval, and the prevalence of prolonged QTc interval gradually increased along with the ADA tertile ascending; secondly, after adjusting for possible risk factors, increased serum ADA levels are notably and positively associated with QTc interval
( $\beta=0.217, t=3.400, P<0.01)$; and thirdly, multivariate regression analysis showed that ADA was a significant risk factor contributing to prolonged QTc interval (OR 1.212, CI 1.094-1.343, $P<0.001)$.

Multiple studies in recent years have shown that measurement of serum ADA levels, a routine in the detection of clinical hepatic function, plays an important role in the assessment of metabolic diseases, inflammatory diseases, cardiovascular diseases and so on. IR is the pathological basis of T2D and its complications. Multiple studies have revealed that serum ADA levels are associated with IR in patients with T2D (19) and in patients with polycystic ovary syndrome (20). The underlying mechanism may be that adenosine, the substrate of ADA, can promote glucose uptake into cells, and increased ADA can reduce

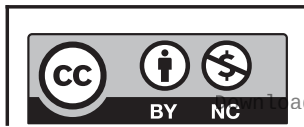

This work is licensed under a Creative Commons Attribution-NonCommercial 4.0 International License. ded from Bioscientifica.com at 04/26/2023 10:29:17AM via free access 




Figure 1

The relationships between serum ADA levels and QTc interval in the participants.

insulin sensitivity through degrading adenosine (21). Correspondingly, treatment with metformin can reduce serum ADA levels in patients with T2D. Thus, ADA may be closely related to type 2 diabetic status.

In addition, serum ADA levels are connected with inflammation level in various inflammatory diseases (22). Inflammation exerts an important role in the development and progression of various diabetic complications. ADA is highly expressed in lymphoid tissues and is essential for the proliferation and differentiation of $\mathrm{T}$ lymphocytes as well as the regulation of the maturation and function of monocytes and macrophages (23). The expression of ADA is significantly increased in tissues rich in macrophage in a rat model of fecal peritonitis (24). Yu et al. revealed that in patients with T2D, serum ADA levels were positively associated with C-reactive protein (CRP) and could be a useful marker to assess the severity of atherosclerosis (14). In our previous study, serum ADA levels were significantly associated with evaluation indexes of diabetic kidney

Table 2 Multiple linear regression analysis to explore the role of ADA to QTc interval.

\begin{tabular}{|c|c|c|c|c|c|}
\hline Models & B $(95 \% \mathrm{Cl})$ & $\beta$ & $\boldsymbol{t}$ & $\boldsymbol{P}$ & $\begin{array}{l}R^{2} \text { for } \\
\text { model }\end{array}$ \\
\hline Model 0 & $2.152(1.616-2.687)$ & 0.336 & 7.892 & $<0.001$ & 0.113 \\
\hline Model 1 & $1.731(0.989-2.473)$ & 0.246 & 4.588 & $<0.001$ & 0.168 \\
\hline Model 2 & $1.488(0.625-2.351)$ & 0.210 & 3.395 & 0.001 & 0.208 \\
\hline Model 3 & $1.537(0.647-2.427)$ & 0.217 & 3.400 & 0.001 & 0.249 \\
\hline
\end{tabular}

Model 0: unadjusted model; Model 1: adjusted for age, male, diabetic duration, BMI; Model 2: additionally adjusted for SBP, DBP, HbA1c, TG, TC, HDL-C, LDL-C, UA, UACR, eGFR; Model 3: additionally adjusted for antidiabetic treatments, antihypertensive treatments, statin medications. disease (DKD), such as UACR, cystatin C and eGFR, and potentially were an important risk factor for DKD. Diabetic retinopathy (DR) is often associated with DKD, and the researchers also observed similar findings that elevated ADA could upregulate the production of cytokines in macrophages, eventually aggravating the breakdown of the blood-retinal barrier (BRB) and the development of DR (25). Hence, ADA may participate in the progression of multiple diabetic complications. In the present study, serum ADA levels were positively corrected with QTc interval and were an independent contributor to prolonged QTc interval.

QTc interval reflects the repolarization of the myocardium, and prolonged QTc interval is a marker of the extension of the vulnerable period, leading to an increased risk of ventricular arrhythmias and sudden cardiac death (SCD) (26). Since ample evidence have fully demonstrated that prolonged QTc interval is closely associated with poor prognosis in patients with T2D, seeking an early intervention of risk factors for prolonged QTc is beneficial to improve the prognosis of these patients. Previous studies have identified clinical parameters, such as aging (27), female (28), hypertension (29), hyperglycemia (7), insulin resistance (29), diabetic microvascular complications (7, $30)$, cardiac autonomic neuropathy (CAN) (15), coronary heart disease (CHD) (15), non-alcoholic fatty liver disease (NAFLD) (31) and use of insulin (29) as potential risk factors for prolonged QTc interval in type 2 diabetic patients. In agreement with previous studies, the present study constructed univariate analysis and demonstrated that aging, female, longer diabetes duration, higher SBP, use of insulin, increased $\mathrm{HbA1c}$ and UACR level were associated with increased risk of prolonged QTc interval. Besides, insulin-sensitizer treatment was also observed to be a risk factor for prolonged QTc interval by univariate and multivariate analysis. Similarly, insulin-sensitizer treatment could induce ischemic ventricular fibrillation by blocking cardiac ATP-sensitive potassium channels in a porcine model with T2D (32). After multivariate logistic regression analysis, only female sex, use of insulinsensitizers and ADA were the independent contributors to prolonged QTc interval. Therefore, serum ADA level may assist clinicians in evaluating the risk of prolonged QTc interval, and even may serve as a potential therapeutic target to ameliorate the prolongation of QTc interval and its associated prognosis in patients with T2D.

Several possible mechanisms linking serum ADA levels and the QTc interval are available. First, serum ADA levels are related to anabatic IR and reduced insulin sensitivity. In experimental animals with T2D, protein expression of Kv4.2 and KChIP2 were reduced by IR, which reduced transient

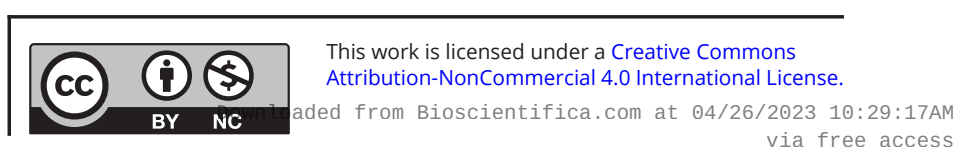


Table 3 Univariate and multivariate analysis to explore relationships between multiple risk factors and prolonged QTc.

\begin{tabular}{l}
\hline Variable \\
\hline Age \\
Female \\
Diabetes duration \\
BMI \\
SBP \\
DBP \\
Insulin treatment \\
Metformin \\
Acarbose \\
Insulin secretagogues \\
Insulin sensitizers \\
DPP-4 inhibitors \\
CCB \\
ARB \\
Diuretics \\
Statin medications \\
HbA1C \\
TG \\
TC \\
HDL-C \\
LDL-C \\
UA \\
eGFR \\
UACR \\
ADA \\
\hline
\end{tabular}

\begin{tabular}{c}
\hline Univariate analysis $(\mathrm{OR} ; 95 \% \mathrm{Cl})$ \\
\hline $1.209(1.011-1.048)$ \\
$3.396(2.188-5.270)$ \\
$1.039(1.012-1.066)$ \\
$1.055(0.990-1.123)$ \\
$1.013(1.002-1.024)$ \\
$0.999(0.983-1.017)$ \\
$1.845(1.178-2.889)$ \\
$0.954(0.622-1.464)$ \\
$0.906(0.436-1.886)$ \\
$1.035(0.659-1.626)$ \\
$2.357(1.133-4.905)$ \\
$0.162(0.021-1.219)$ \\
$1.149(0.703-1.878)$ \\
$1.542(0.939-2.533)$ \\
$1.947(0.931-4.071)$ \\
$0.702(0.234-2.107)$ \\
$1.173(1.062-1.295)$ \\
$1.026(0.954-1.104)$ \\
$1.079(0.912-1.277)$ \\
$1.123(0.508-2.482)$ \\
$1.036(0.821-1.305)$ \\
$1.002(1.000-1.004)$ \\
$0.993(0.986-1.001)$ \\
$1.001(1.000-1.001)$ \\
$1.224(1.153-1.299)$ \\
\end{tabular}

$\begin{array}{r}\hline \multicolumn{1}{c}{\boldsymbol{P}} \\ \hline 0.002 \\ <0.001 \\ 0.004 \\ 0.099 \\ 0.022 \\ 0.943 \\ 0.007 \\ 0.954 \\ 0.792 \\ 0.882 \\ 0.022 \\ 0.077 \\ 0.579 \\ 0.087 \\ 0.077 \\ 0.528 \\ 0.002 \\ 0.492 \\ 0.375 \\ 0.775 \\ 0.768 \\ 0.109 \\ 0.080 \\ 0.006 \\ <0.001 \\ \hline\end{array}$

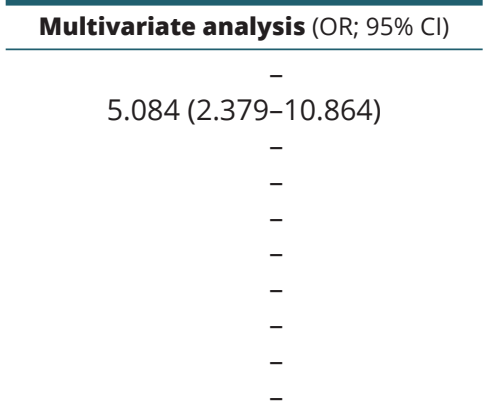

$4.229(1.290-13.860)$

0.017



$-$

$-$

$-$

$-$

$-$

$-$

0.768

.109

0.006

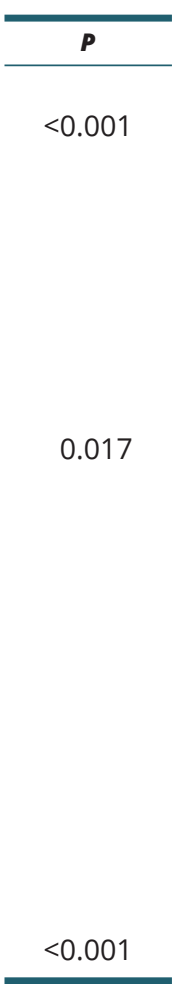

Results are given as odds ratios and $95 \% \mathrm{Cls}(\mathrm{OR} ; 95 \% \mathrm{Cl})$. Nagelkerke $\mathrm{R}^{2}=0.371$ in multivariate analysis.

outward $\mathrm{K}^{+}$current and resulted in the prolongation of action potential duration and QT interval (33). Moreover, IR and associated hyperinsulinemia may contribute to left ventricular hypertrophy, and the consequent increased left ventricular mass is a strong determinant of QT interval in type 2 diabetic patients (34). Secondly, ADA is closely associated with inflammation in both in vivo and in vitro studies. In a cross-sectional study, inflammatory markers CRP and tumor necrosis factor receptor 1 were associated with QTC prolongation (35). In vitro studies have shown that inflammation can cause APD prolongation and arrhythmias by inducing calcium leakage from the sarcoplasmic reticulum (36). Thirdly, serum ADA levels may reflect the severity of chronic myocardial ischemia in patients with T2D. Peters et al. (37) demonstrated that QTc prolongation was observed 5-21 days after acute myocardial infarction. Dekker et al. (38) also found that QTc interval was associated with chronic myocardial ischemia in apparently healthy population. In patients with chronic myocardial ischemia, successful revascularization can significantly improve QT interval prolongation (39). Therefore, serum ADA level may be a potential marker for predicting prolonged QTc interval.

It should be pointed out that our study had some limitations. First, as the present study is a cross-sectional study, the cause-effect relationship between high serum
ADA levels and QTc prolongation cannot be fully proved. Secondly, all the subjects enrolled in this study were Chinese, which limited the generalizability of our study. Thirdly, adenosine is the substrate of ADA and exerts multiple functions, but this study did not simultaneously detect adenosine levels. Hereby, further research should be conducted to validate the results of our study and to address the above limitations.

\section{Conclusions}

In conclusion, serum ADA levels were independently associated with prolonged QTc interval in type 2 diabetic patients. In clinical practice, the level of ADA can be obtained at the time of hepatic function measurement without additional burden to patients and can help clinicians stratify cardiovascular risk in patients with T2D.

\section{Declaration of interest}

The authors declare that there is no conflict of interest that could be perceived as prejudicing the impartiality of the research reported.

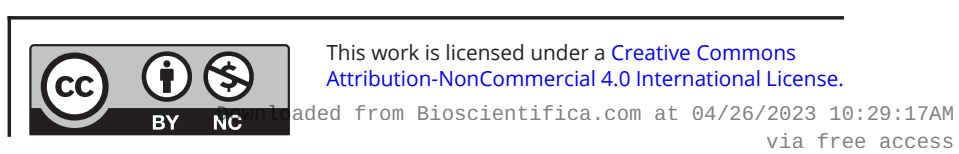




\section{Funding}

The study was supported by the Medical Research Project of Health Commission of Nantong (MB2020012, WKZL2018041).

\section{Ethics approval and consent to participate}

The study was approved by the institutional review board of Affiliated Hospital 2 of Nantong University and First People's Hospital of Nantong City, and written informed consent was obtained from all participants.

\section{Availability of data and materials}

The current data are available to all interested researchers upon reasonable request. Requests for access to data should be made to the principal investigators of the study.

\section{Author contribution statement}

$C L, X G$ and X W participated in the design of the study, data collection, analysis of the data, and drafting of the manuscript. J $S$ and $C L$ conceived of the study, participated in its design and revised the manuscript. $C L$ and $Y$ W participated in the analysis of the data and revised the manuscript. $C$ $L, D Z, F X, W$ L and M S participated in data collection. All authors read and approved the final manuscript.

\section{References}

1 Holman N, Young B \& Gadsby R. Current prevalence of Type 1 and Type 2 diabetes in adults and children in the UK. Diabetic Medicine 201532 1119-1120. (https://doi.org/10.1111/dme.12791)

2 Zheng Y, Ley SH \& Hu FB. Global aetiology and epidemiology of type 2 diabetes mellitus and its complications. Nature Reviews: Endocrinology 201814 88-98. (https://doi.org/10.1038/nrendo.2017.151)

3 Svensson AK, Svensson T, Kitlinski M, Almgren P, Engström G, Nilsson PM \& Melander O. Incident diabetes mellitus may explain the association between sleep duration and incident coronary heart disease. Diabetologia 2018 61 331-341. (https://doi.org/10.1007/s00125-017-4464-3)

4 Yetkin DO, Kucukkaya B, Turhan M \& Oren M. The effect of 25-hydroxyvitamin D levels on QT interval duration and dispersion in type 2 diabetic patients. Croatian Medical Journal 201556 525-530. (https://doi.org/10.3325/cmj.2015.56.525)

5 Cox AJ, Azeem A, Yeboah J, Soliman EZ, Aggarwal SR, Bertoni AG, Carr JJ, Freedman BI, Herrington DM \& Bowden DW. Heart ratecorrected QT interval is an independent predictor of all-cause and cardiovascular mortality in individuals with type 2 diabetes: the Diabetes Heart Study. Diabetes Care 201437 1454-1461. (https://doi. org/10.2337/dc13-1257)

6 Elming H, Holm E, Jun L, Torp-Pedersen C, Køber L, Kircshoff M, Malik M \& Camm J. The prognostic value of the QT interval and QT interval dispersion in all-cause and cardiac mortality and morbidity in a population of Danish citizens. European Heart Journal 199819 1391-1400. (https://doi.org/10.1053/euhj.1998.1094)

7 Li X, Ren H, Xu ZR, Liu YJ, Yang XP \& Liu JQ. Prevalence and risk factors of prolonged QTc interval among Chinese patients with type 2 diabetes. Experimental Diabetes Research 20122012 234084. (https:// doi.org/10.1155/2012/234084)

8 Niraula A, Thapa S, Kunwar S, Lamsal M, Baral N \& Maskey R. Adenosine deaminase activity in type 2 diabetes mellitus: does it have any role? BMC Endocrine Disorders 201818 58. (https://doi.org/10.1186/ s12902-018-0284-9)

9 Kurtul N, Pence S, Akarsu E, Kocoglu H, Aksoy Y \& Aksoy H. Adenosine deaminase activity in the serum of type 2 diabetic patients. Acta Medica 200447 33-35. (https://doi.org/10.14712/18059694.2018.63)
10 Mokhtari M, Hashemi M, Yaghmaei M, Molashahi F, Shikhzadeh A, Niazi A \& Ghavami S. Serum adenosine deaminase activity in gestational diabetes mellitus and normal pregnancy. Archives of Gynecology and Obstetrics 2010281 623-626. (https://doi.org/10.1007/ s00404-009-1148-3)

11 Sapkota LB, Thapa S \& Subedi N. Correlation study of adenosine deaminase and its isoenzymes in type 2 diabetes mellitus. BMJ Open Diabetes Research and Care 20175 e000357. (https://doi.org/10.1136/ bmjdrc-2016-000357)

12 Gewirtz H, Brautigan DL, Olsson RA, Brown P \& Most AS. Role of adenosine in the maintenance of coronary vasodilation distal to a severe coronary artery stenosis. Observations in conscious domestic swine. Circulation Research 198353 42-51. (https://doi.org/10.1161/01. res.53.1.42)

13 Shryock JC \& Belardinelli L. Adenosine and adenosine receptors in the cardiovascular system: biochemistry, physiology, and pharmacology. American Journal of Cardiology 199779 2-10. (https://doi.org/10.1016/ s0002-9149(97)00256-7)

14 Yu M, Zhou H, Li Q, Ding J, Shuai H \& Zhang J. Serum adenosine deaminase as a useful marker to estimate coronary artery calcification in type 2 diabetes mellitus patients. Clinical and Applied Thrombosis/Hemostasis 202127 1076029621999722. (https://doi. org/10.1177/1076029621999722)

15 Ninkovic VM, Ninkovic SM, Miloradovic V, Stanojevic D, Babic M, Giga V, Dobric M, Trenell MI, Lalic N, Seferovic PM, et al. Prevalence and risk factors for prolonged QT interval and QT dispersion in patients with type 2 diabetes. Acta Diabetologica 201653 737-744. (https://doi.org/10.1007/s00592-016-0864-y)

16 American Diabetes Association. Diagnosis and classification of diabetes mellitus. Diabetes Care 201336 (Supplement 1) S67-S74. (https://doi.org/10.2337/dc13-S067)

17 Salles GF, Cardoso CR \& Deccache W. Multivariate associates of QT interval parameters in diabetic patients with arterial hypertension: importance of left ventricular mass and geometric patterns. Journal of Human Hypertension 200317 561-567. (https://doi.org/10.1038/ sj.jhh.1001590)

18 Inker LA, Schmid CH, Tighiouart H, Eckfeldt JH, Feldman HI, Greene T, Kusek JW, Manzi J, Van Lente F, Zhang YL, et al. Estimating glomerular filtration rate from serum creatinine and cystatin C. New England Journal of Medicine 2012367 20-29. (https://doi.org/10.1056/ NEJMoa1114248)

19 Koopmans SJ, Sips HC, Bosman J, Radder JK \& Krans HM. Antilipolytic action of insulin in adipocytes from starved and diabetic rats during adenosine-controlled incubations. Endocrinology 1989125 3044-3050. (https://doi.org/10.1210/endo-125-6-3044)

20 Öztürk B, Gürbüz AS, Durak ZE \& Öztürk HS. Dipeptidyl peptidase-4 and adenosine deaminase enzyme levels in polycystic ovary syndrome. Gynecological Endocrinology 201935 138-141. (https://doi. org/10.1080/09513590.2018.1505847)

21 Kather H. Pathways of purine metabolism in human adipocytes. Further evidence against a role of adenosine as an endogenous regulator of human fat cell function. Journal of Biological Chemistry 1990265 96-102. (https://doi.org/10.1016/S0021-9258(19)40200-7)

22 Murray JL, Loftin KC, Munn CG, Reuben JM, Mansell PW \& Hersh EM. Elevated adenosine deaminase and purine nucleoside phosphorylase activity in peripheral blood null lymphocytes from patients with acquired immune deficiency syndrome. Blood 198565 1318-1324. (https://doi.org/10.1182/blood.V65.6.1318.bloodjournal6561318)

23 Xuan C, Tian QW, Zhang SY, Li H, Tian TT, Zhao P, Yue K, Ling YY, He GW \& Lun LM. Serum adenosine deaminase activity and coronary artery disease: a retrospective case-control study based on 9929 participants. Therapeutic Advances in Chronic Disease 201910 2040622319891539. (https://doi.org/10.1177/2040622319891539)

24 Law WR, Valli VE \& Conlon BA. Therapeutic potential for transient inhibition of adenosine deaminase in systemic inflammatory response syndrome. Critical Care Medicine 200331 1475-1481. (https://doi. org/10.1097/01.CCM.0000063259.09580.D8) 
25 Samra YA, Saleh HM, Hussein KA, Elsherbiny NM, Ibrahim AS, Elmasry K, Fulzele S, El-Shishtawy MM, Eissa LA, Al-Shabrawey M, et al. Adenosine deaminase-2-induced hyperpermeability in human retinal vascular endothelial cells is suppressed by microRNA-146b-3p. Investigative Ophthalmology and Visual Science 201758 933-943. (https://doi.org/10.1167/iovs.16-19782)

26 Rautaharju PM, Surawicz B, Gettes LS, Bailey JJ, Childers R, Deal BJ, Gorgels A, Hancock EW, Josephson M, Kligfield P, et al. AHA/ACCF/ HRS recommendations for the standardization and interpretation of the electrocardiogram: Part IV: the ST segment, T and U waves, and the QT interval: a scientific statement from the American Heart Association Electrocardiography and Arrhythmias Committee, Council on Clinical Cardiology; the American College of Cardiology Foundation; and the Heart Rhythm Society. Endorsed by the International Society for Computerized Electrocardiology. Journal of the American College of Cardiology 200953 982-991. (https://doi. org/10.1016/j.jacc.2008.12.014)

27 Mangoni AA, Kinirons MT, Swift CG \& Jackson SH. Impact of age on QT interval and QT dispersion in healthy subjects: a regression analysis. Age and Ageing 200332 326-331. (https://doi.org/10.1093/ageing/32.3.326)

28 Stramba-Badiale M, Locati EH, Martinelli A, Courville J \& Schwartz PJ. Gender and the relationship between ventricular repolarization and cardiac cycle length during 24-h Holter recordings. European Heart Journal 199718 1000-1006. (https://doi.org/10.1093/oxfordjournals. eurheartj.a015357)

29 Yang XH, Su JB, Zhang XL, Zhao LH, Xu F, Wang XQ \& Cheng XB. The relationship between insulin sensitivity and heart rate-corrected QT interval in patients with type 2 diabetes. Diabetology and Metabolic Syndrome 20179 69. (https://doi.org/10.1186/s13098-017-0268-3)

30 Subbalakshmi NK, Adhikari PM, Sathyanarayana Rao KN \& Jeganathan PS. Influencing factors of QTc among the clinical characteristics in type 2 diabetes mellitus. Diabetes Research and Clinical Practice 201088 265-272. (https://doi.org/10.1016/j. diabres.2010.03.001)

31 Hung CS, Tseng PH, Tu CH, Chen CC, Liao WC, Lee YC, Chiu HM, Lin HJ, Ho YL, Yang WS, et al. Nonalcoholic fatty liver disease is associated with QT prolongation in the general population. Journal of the American Heart Association 20154 e001820. (https://doi.org/10.1161/ JAHA.115.001820)
32 Sarraf M, Lu L, Ye S, Reiter MJ, Greyson CR \& Schwartz GG. Thiazolidinedione drugs promote onset, alter characteristics, and increase mortality of ischemic ventricular fibrillation in pigs. Cardiovascular Drugs and Therapy 201226 195-204. (https://doi. org/10.1007/s10557-012-6384-2)

33 Sato T, Kobayashi T, Kuno A, Miki T, Tanno M, Kouzu H, Itoh T, Ishikawa S, Kojima T, Miura T, et al. Type 2 diabetes induces subendocardium-predominant reduction in transient outward $\mathrm{K}+$ current with downregulation of Kv4.2 and KChIP2. American Journal of Physiology: Heart and Circulatory Physiology 2014306 H1054-H1065. (https://doi.org/10.1152/ajpheart.00414.2013)

34 Dawson A, Morris AD \& Struthers AD. The epidemiology of left ventricular hypertrophy in type 2 diabetes mellitus. Diabetologia 2005 48 1971-1979. (https://doi.org/10.1007/s00125-005-1896-y)

35 Medenwald D, Kors JA, Loppnow H, Thiery J, Kluttig A, Nuding S, Tiller D, Greiser KH, Werdan K \& Haerting J. Inflammation and prolonged QT time: results from the Cardiovascular Disease, Living and Ageing in Halle (CARLA) study. PLoS ONE 20149 e95994. (https:// doi.org/10.1371/journal.pone.0095994)

36 Abramochkin DV, Kuzmin VS, Mitrochin VM, Kalugin L, Dvorzhak A, Makarenko EY, Schubert R \& Kamkin A. TNF- $\alpha$ provokes electrical abnormalities in rat atrial myocardium via a NO-dependent mechanism. Pflugers Archiv 2013465 1741-1752. (https://doi. org/10.1007/s00424-013-1320-2)

37 Peters RW, Byington RP, Barker A \& Yusuf S. Prognostic value of prolonged ventricular repolarization following myocardial infarction: the Bhat experience. The BHAT Study Group. Journal of Clinical Epidemiology 199043 167-172. (https://doi.org/10.1016/08954356(90)90180-w)

38 Dekker JM, Crow RS, Hannan PJ, Schouten EG, Folsom AR \& ARIC Study. Heart rate-corrected QT interval prolongation predicts risk of coronary heart disease in black and white middle-aged men and women: the ARIC study. Journal of the American College of Cardiology 200443 565-571. (https://doi.org/10.1016/j.jacc.2003.09.040)

39 Mirbolouk F, Arami S, Salari A, Shad B, Kazemnejad E \& Moladoust H. Corrected QT-interval and dispersion after revascularization by percutaneous coronary intervention and coronary artery bypass graft surgery in chronic ischemia. Journal of Invasive Cardiology 201426 444-450.

Received in final form 18 June 2021

Accepted 14 July 2021

Accepted Manuscript published online 14 July 2021
This work is licensed under a Creative Commons Attribution-NonCommercial 4.0 International License. ded from Bioscientifica.com at 04/26/2023 10:29:17AM 